\title{
A Metabolomics Analysis of Adiposity and Advanced Prostate Cancer Risk in the Health Professionals Follow-Up Study
}

\author{
Barbra A. Dickerman ${ }^{1, *}$, Ericka M. Ebot ${ }^{1}$, Brian C. Healy ${ }^{2,3,4}$, Kathryn M. Wilson ${ }^{1,5}$, \\ A. Heather Eliassen ${ }^{1,5}{ }^{-}$, Alberto Ascherio ${ }^{1,5,6}$, Claire H. Pernar ${ }^{1}$, Oana A. Zeleznik ${ }^{5}{ }^{\oplus}$, \\ Matthew G. Vander Heiden ${ }^{7,8}$ D, Clary B. Clish ${ }^{9}$, Edward Giovannucci ${ }^{1,5,6}$ and \\ Lorelei A. Mucci 1,5 \\ 1 Department of Epidemiology, Harvard T.H. Chan School of Public Health, Boston, MA 02115, USA; \\ enoonan@hsph.harvard.edu (E.M.E.); kwilson@hsph.harvard.edu (K.M.W.); \\ nhahe@channing.harvard.edu (A.H.E.); aascheri@hsph.harvard.edu (A.A.); \\ cpernar@mail.harvard.edu (C.H.P.); egiovann@hsph.harvard.edu (E.G.); lmucci@hsph.harvard.edu (L.A.M.) \\ Department of Neurology, Harvard Medical School, Boston, MA 02115, USA; bchealy@mgh.harvard.edu \\ Partners Multiple Sclerosis Center, Brigham and Women's Hospital, Boston, MA 02115, USA \\ Biostatistics Center, Massachusetts General Hospital, Boston, MA 02114, USA \\ 5 Channing Division of Network Medicine, Department of Medicine, Brigham and Women's Hospital and \\ Harvard Medical School, Boston, MA 02115, USA; nhotz@channing.harvard.edu \\ 6 Department of Nutrition, Harvard T.H. Chan School of Public Health, Boston, MA 02115, USA \\ 7 Koch Institute for Integrative Cancer Research and Department of Biology, Massachusetts Institute of \\ Technology, Cambridge, MA 02139, USA; mvh@mit.edu \\ 8 Department of Medical Oncology, Dana-Farber Cancer Institute, Boston, MA 02215, USA \\ 9 Broad Institute of Massachusetts, Institute of Technology and Harvard, Boston, MA 02142, USA; \\ clary@broadinstitute.org \\ * Correspondence: bad788@mail.harvard.edu
}

Received: 10 January 2020; Accepted: 7 March 2020; Published: 10 March 2020

\begin{abstract}
Obesity is associated with a higher risk of advanced prostate cancer, but men with the same body mass index (BMI) may differ in their underlying metabolic health. Using metabolomics data from nested case-control studies in the Health Professionals Follow-Up Study, we calculated Pearson correlations between 165 circulating metabolites and three adiposity measures (BMI, waist circumference, and derived fat mass from a validated prediction equation) to identify adiposity-associated metabolites. We used Lasso to further select metabolites for prediction models of adiposity measures, which we used to calculate metabolic scores representing metabolic obesity. In an independent set of 212 advanced prostate cancer cases (T3b/T4/N1/M1 or lethal during follow-up) and 212 controls, we used logistic regression to evaluate the associations between adiposity measures and metabolic scores with risk of advanced disease. All adiposity measures were associated with higher blood levels of carnitines (Pearson $r$ range, 0.16 to 0.18$)$ and lower levels of glutamine $(r=-0.19)$ and glycine $(r,-0.29$ to -0.20$)$, in addition to alterations in various lipids. No adiposity measure or metabolic score was associated with risk of advanced prostate cancer (e.g., odds ratio for a $5 \mathrm{~kg} / \mathrm{m}^{2}$ increase in BMI 0.96 (95\% CI: 0.73, 1.27) and BMI metabolic score 1.18 (95\% CI: 0.57, 2.48)). BMI, waist circumference, and derived fat mass were associated with a broad range of metabolic alterations. Neither adiposity nor metabolic scores were associated with risk of advanced prostate cancer.
\end{abstract}

Keywords: adiposity; epidemiology; fat mass; metabolomics; obesity; advanced prostate cancer; waist circumference 


\section{Introduction}

Obesity is associated with a higher risk of advanced prostate cancer [1]. However, men with the same body mass index (BMI) may differ in their underlying metabolic health and subsequent disease risk [2-5]. The integration of metabolomics and anthropometric data offers the potential to better identify men at highest risk of prostate cancer, elucidate underlying mechanisms, and inform the development of targeted intervention strategies.

Previous metabolomics-based studies have identified the metabolic alterations associated with BMI and waist circumference [4,6-10], but few have evaluated fat mass [11,12]. Some of these studies further examined how the identified metabolites individually related to later risk of diabetes and breast cancer [6,8]. No study to date has examined whether obesity-related metabolic alterations could, collectively as a metabolic signature of obesity, facilitate the identification of men at risk of advanced prostate cancer by adding information beyond standard measures of obesity, such as BMI.

Here we evaluate associations between metabolites, adiposity, and advanced prostate cancer. First, we identify the plasma metabolites associated with various adiposity measures (BMI, waist circumference, and derived fat mass). Second, we use selected metabolites to evaluate the association between metabolically defined obesity and risk of advanced prostate cancer, among all men and within subgroups defined by self-reported adiposity measures.

\section{Results}

\subsection{Population Characteristics}

Table 1 shows the baseline characteristics of the 660 eligible men. The mean age at blood draw was 65 years, the mean BMI was $26 \mathrm{~kg} / \mathrm{m}^{2}$, the mean waist circumference was $96 \mathrm{~cm}$, and the mean derived fat mass was $22 \mathrm{~kg}$. Controls in the Parkinson's disease study were more likely to be former smokers compared with men in the amyotrophic lateral sclerosis (ALS) and prostate cancer studies. Advanced prostate cancer cases were more likely to be current smokers than their matched controls.

Table 1. Baseline characteristics of eligible participants from nested-case control studies of various outcomes, Health Professionals Follow-Up Study, 1993-1996 a .

\begin{tabular}{|c|c|c|c|c|}
\hline \multirow{2}{*}{ Characteristic, Mean (SD) or \% } & \multirow{2}{*}{$\begin{array}{l}\text { Amyotrophic Lateral } \\
\text { Sclerosis Study } \\
\text { Controls } \\
(n=52)\end{array}$} & \multirow{2}{*}{$\begin{array}{c}\begin{array}{c}\text { Parkinson's } \\
\text { Disease Study }\end{array} \\
\text { Controls } \\
(n=184)\end{array}$} & \multicolumn{2}{|c|}{ Prostate Cancer Study } \\
\hline & & & $\begin{array}{l}\text { Controls } \\
(n=212)\end{array}$ & $\begin{array}{c}\text { Advanced Cases } \\
\quad(n=212)\end{array}$ \\
\hline Age (years) & $62.7(8.3)$ & $65.3(8.0)$ & $65.3(8.4)$ & $65.4(8.5)$ \\
\hline Body mass index $\left(\mathrm{kg} / \mathrm{m}^{2}\right)$ & $25.9(2.5)$ & $25.5(2.8)$ & $25.8(3.6)$ & $25.8(4.1)$ \\
\hline Waist circumference $(\mathrm{cm})$ & $94.7(8.1)$ & $95.5(8.5)$ & $96.5(10.3)$ & $95.7(9.8)$ \\
\hline Derived fat mass $(\mathrm{kg})^{\mathrm{b}}$ & $21.9(4.3)$ & $21.6(5.2)$ & $22.5(6.4)$ & $21.7(5.9)$ \\
\hline Total physical activity (MET hours/week) & $39.7(29.3)$ & $34.4(27.4)$ & $32.5(25.6)$ & $31.1(28.9)$ \\
\hline \multicolumn{5}{|l|}{ Year of blood donation } \\
\hline 1993-1994 & 87 & 94 & 97 & 97 \\
\hline 1995-1996 & 14 & 6 & 3 & 3 \\
\hline Fasting $\geq 8 \mathrm{~h}$ & 50 & 55 & 62 & 67 \\
\hline Race/ethnicity, White & 94 & 98 & 97 & 99 \\
\hline \multicolumn{5}{|l|}{ Smoking status } \\
\hline Never & 50 & 39 & 46 & 43 \\
\hline Former & 46 & 58 & 50 & 51 \\
\hline Current & 4 & 3 & 4 & 7 \\
\hline History of diabetes mellitus & 4 & 4 & 6 & 7 \\
\hline Family history of prostate cancer & - & - & 9 & 9 \\
\hline Recent prostate-specific antigen testing ${ }^{c}$ & - & - & 60 & 62 \\
\hline
\end{tabular}

Abbreviation: MET, Metabolic equivalent of task. Frequencies of polytomous variables may not add up to $100 \%$ due to rounding. ${ }^{a}$ Baseline characteristics were measured at the time of blood draw when possible, and otherwise in the questionnaire preceding blood draw. ${ }^{b}$ Derived fat mass from validated anthropometric prediction models developed in the National Health and Nutrition Examination Survey (NHANES). ${ }^{c}$ Prostate-specific antigen testing since January 1, 1992. 
The mean age at advanced prostate cancer diagnosis was 72 years (range, 50-92). Among the 212 men with advanced prostate cancer, 53\% had T3b/T4/N1/M1 tumors at diagnosis and the remaining $47 \%$ were classified as such because of metastasis or fatal disease. Among the $88 \%$ of cases for whom the grade was available, $37 \%$ had high-grade tumors (Gleason grade $\geq 8$ ). Among the $85 \%$ of cases for whom prostate-specific antigen (PSA) level at diagnosis was available, the median PSA was $8.5 \mathrm{ng} / \mathrm{mL}$. The median time between the blood draw and prostate cancer diagnosis was 5.5 years (range, 0.1-16.2).

\subsection{Metabolites Associated with Adiposity}

We identified 43 metabolites associated (false discovery rate (FDR) $p$-value $<0.05$ and $|\mathrm{r}| \geq 0.15$ ) with BMI, 38 with waist circumference, and 33 with derived fat mass (Table 2). There was considerable overlap in identified metabolites across adiposity measures; 28 metabolites were associated with all three measures (in the same direction) (Figure S3). All adiposity measures were associated with higher levels of carnitines ( $r, 0.16$ to 0.18 ) and specific diacylglycerol (DAG) and triacylglycerol (TAG) lipid species ( $r, 0.16$ to 0.24$)$. All were associated with lower levels of glycine ( $r,-0.29$ to -0.20$)$, glutamine $(r=-0.19)$, as well as some cholesterol ester (CE) ( $r,-0.23$ to -0.19$)$, lysophosphatidylcholine (LPC) ( $r,-0.34$ to -0.18$)$, and lysophosphatidylethanolamine (LPE) (r, -0.29 to -0.17$)$. Mixed associations were found for phosphatidylcholine (PC) (r, -0.18 to 0.20$)$.

Associations were similar when restricted to men with information on all three adiposity measures (Table S1). When restricted to fasting samples, only BMI and derived fat mass were associated with lower glycine $(r,-0.32$ to -0.31$)$ and LPE ( $r,-0.26$ to -0.25$)$, BMI was associated with higher valine $(r=0.23)$, waist circumference was associated with higher ceramides ( $r, 0.23$ to 0.24$)$, all adiposity measures were associated with higher PC (r, 0.25 to 0.28$)$, and neither glutamine nor carnitines were identified as adiposity-associated metabolites; patterns of associations were otherwise similar, with stronger correlations observed for various DAG and TAG lipid species (r, 0.24 to 0.36) (Table S2).

In Lasso models to derive metabolic scores, there were 15 metabolites with a non-zero coefficient in models for BMI, 15 for waist circumference, and 21 for derived fat mass (File S2). Each model retained at least one of the identified amino acids and the top hit for carnitines, LPC, LPE, and PC, in addition to a selection of lipids. 
Table 2. Metabolites associated with adiposity measures (FDR $p$-value $<0.05$ and $|r| \geq 0.15$ ) among controls $\left(n=219-234\right.$ ) ${ }^{\text {a }}$ from nested case-control studies, Health Professionals Follow-Up Study, 1993-1996.

\begin{tabular}{|c|c|c|c|c|c|c|c|}
\hline \multirow{2}{*}{ HMDB ID ${ }^{b}$} & \multirow{2}{*}{ Metabolite Name } & \multicolumn{2}{|c|}{ Body Mass Index } & \multicolumn{2}{|c|}{ Waist Circumference } & \multicolumn{2}{|c|}{ Derived Fat Mass } \\
\hline & & $\begin{array}{l}\text { Pearson Correlation } \\
\text { Coefficient }^{c}\end{array}$ & $\begin{array}{c}\text { FDR } \\
p \text {-value }\end{array}$ & $\begin{array}{l}\text { Pearson Correlation } \\
\text { Coefficient }^{c}\end{array}$ & $\begin{array}{c}\text { FDR } \\
p \text {-value }\end{array}$ & $\begin{array}{l}\text { Pearson Correlation } \\
\text { Coefficient }^{c}\end{array}$ & $\begin{array}{c}\text { FDR } \\
p \text {-value }\end{array}$ \\
\hline Amino acids & & & & & & & \\
\hline HMDB00123 & glycine & -0.29 & $<0.001$ & -0.20 & 0.02 & -0.25 & 0.01 \\
\hline $\begin{array}{l}\text { HMDB00641 } \\
\text { Carnitines }\end{array}$ & glutamine & -0.19 & 0.02 & -0.19 & 0.03 & -0.19 & 0.04 \\
\hline HMDB06347 & C26 carnitine & 0.18 & 0.03 & 0.18 & 0.04 & - & - \\
\hline HMDB00688 & C5 carnitine & 0.18 & 0.03 & - & - & - & - \\
\hline HMDB00705 & C6 carnitine & 0.18 & 0.03 & 0.18 & 0.03 & - & - \\
\hline HMDB02013 & C4 carnitine & 0.16 & 0.05 & - & - & - & - \\
\hline HMDB13326 & C12:1 carnitine & - & - & 0.17 & 0.04 & - & - \\
\hline $\begin{array}{l}\text { HMDB00222 } \\
\text { Lipids } \\
\text { CE }\end{array}$ & C16 carnitine & - & - & - & - & 0.18 & 0.04 \\
\hline HMDB10375 & C22:5 CE & -0.20 & 0.01 & -0.22 & 0.01 & -0.23 & 0.01 \\
\hline $\begin{array}{l}\text { HMDB06733 } \\
\text { DAG }\end{array}$ & C22:6 CE & - & - & - & - & -0.19 & 0.03 \\
\hline $\begin{array}{c}\text { Saturated } \\
\text { HMDB07098 } \\
\text { Unsaturated }\end{array}$ & C32:0 DAG & 0.23 & 0.01 & 0.21 & 0.01 & 0.22 & 0.01 \\
\hline HMDB07102 & C34:1 DAG & 0.24 & $<0.01$ & 0.24 & 0.01 & 0.24 & 0.01 \\
\hline HMDB07099 & C32:1 DAG & 0.23 & 0.01 & 0.24 & 0.01 & 0.24 & 0.01 \\
\hline HMDB07103 & C34:2 DAG & 0.22 & 0.01 & 0.23 & 0.01 & 0.23 & 0.01 \\
\hline HMDB07132 & C34:3 DAG & 0.22 & 0.01 & 0.23 & 0.01 & 0.22 & 0.01 \\
\hline HMDB07218 & C36:2 DAG & 0.21 & 0.01 & 0.23 & 0.01 & 0.22 & 0.01 \\
\hline HMDB07216 & C36:1 DAG & 0.21 & 0.01 & 0.22 & 0.01 & 0.23 & 0.01 \\
\hline HMDB07219 & C36:3 DAG & 0.20 & 0.02 & 0.21 & 0.01 & 0.20 & 0.02 \\
\hline HMDB07248 & C36:4 DAG & 0.17 & 0.04 & 0.17 & 0.04 & - & - \\
\hline $\begin{array}{l}\text { HMDB07199 } \\
\text { LPC }\end{array}$ & C38:5 DAG & 0.16 & 0.05 & - & - & - & - \\
\hline HMDB10386 & C18:2 LPC & -0.34 & $<0.0001$ & -0.23 & 0.01 & -0.29 & $<0.001$ \\
\hline HMDB10397 & C20:5 LPC & -0.34 & $<0.0001$ & -0.24 & 0.01 & -0.29 & $<0.001$ \\
\hline HMDB02815 & C18:1 LPC & -0.29 & $<0.001$ & -0.21 & 0.01 & -0.26 & 0.01 \\
\hline HMDB10404 & C22:6 LPC & -0.25 & $<0.01$ & -0.18 & 0.03 & -0.26 & 0.01 \\
\hline
\end{tabular}


Table 2. Cont.

\begin{tabular}{|c|c|c|c|c|c|c|c|}
\hline \multirow{2}{*}{ HMDB ID $^{b}$} & \multirow{2}{*}{ Metabolite Name } & \multicolumn{2}{|c|}{ Body Mass Index } & \multicolumn{2}{|c|}{ Waist Circumference } & \multicolumn{2}{|c|}{ Derived Fat Mass } \\
\hline & & $\begin{array}{l}\text { Pearson Correlation } \\
\text { Coefficient }^{c}\end{array}$ & $\begin{array}{c}\text { FDR } \\
p \text {-value }\end{array}$ & $\begin{array}{l}\text { Pearson Correlation } \\
\text { Coefficient }^{c}\end{array}$ & $\begin{array}{c}\text { FDR } \\
p \text {-value }\end{array}$ & $\begin{array}{l}\text { Pearson Correlation } \\
\text { Coefficient }^{c}\end{array}$ & $\begin{array}{c}\text { FDR } \\
p \text {-value }\end{array}$ \\
\hline \multicolumn{8}{|l|}{ LPE } \\
\hline HMDB11503 & C16:0 LPE & -0.29 & $<0.001$ & -0.17 & 0.04 & -0.23 & 0.01 \\
\hline HMDB11507 & C18:2 LPE & -0.26 & $<0.01$ & - & - & - & - \\
\hline HMDB11506 & C18:1 LPE & -0.22 & 0.01 & - & - & -0.18 & 0.04 \\
\hline HMDB11130 & C18:0 LPE & -0.20 & 0.02 & - & - & - & - \\
\hline $\begin{array}{c}\text { HMDB11526 } \\
\text { PC }\end{array}$ & C22:6 LPE & -0.19 & 0.02 & - & - & - & - \\
\hline HMDB11210 & $\begin{array}{c}\text { C34:2 PC } \\
\text { plasmalogen }\end{array}$ & -0.16 & 0.05 & - & - & - & - \\
\hline HMDB08047 & C38:3 PC & 0.20 & 0.01 & 0.20 & 0.02 & - & - \\
\hline HMDB08057 & C40:6 PC & 0.19 & 0.02 & 0.19 & 0.03 & - & - \\
\hline $\begin{array}{c}\text { HMDB } 08511 \\
\text { TAG }\end{array}$ & C40:10 PC & -0.16 & 0.05 & - & - & -0.18 & 0.04 \\
\hline Unsaturated & & & & & & & \\
\hline HMDB05369 & C52:2 TAG & 0.23 & 0.01 & 0.23 & 0.01 & 0.23 & 0.01 \\
\hline HMDB05360 & C50:1 TAG & 0.23 & 0.01 & 0.21 & 0.01 & 0.22 & 0.01 \\
\hline HMDB05384 & C52:3 TAG & 0.22 & 0.01 & 0.22 & 0.01 & 0.21 & 0.02 \\
\hline HMDB05433 & C50:3 TAG & 0.22 & 0.01 & 0.23 & 0.01 & 0.22 & 0.01 \\
\hline HMDB05377 & C50:2 TAG & 0.22 & 0.01 & 0.21 & 0.01 & 0.22 & 0.01 \\
\hline HMDB05367 & C52:1 TAG & 0.20 & 0.01 & 0.20 & 0.02 & 0.21 & 0.01 \\
\hline HMDB05376 & C48:2 TAG & 0.19 & 0.02 & 0.20 & 0.02 & 0.21 & 0.02 \\
\hline HMDB05432 & C48:3 TAG & 0.18 & 0.03 & 0.20 & 0.02 & 0.20 & 0.02 \\
\hline HMDB10412 & C46:1 TAG & 0.18 & 0.03 & 0.17 & 0.04 & 0.20 & 0.02 \\
\hline HMDB05403 & C54:2 TAG & 0.18 & 0.03 & 0.20 & 0.02 & 0.20 & 0.02 \\
\hline HMDB05363 & C52:4 TAG & 0.18 & 0.03 & 0.18 & 0.03 & - & - \\
\hline HMDB05359 & C48:1 TAG & 0.17 & 0.04 & 0.17 & 0.04 & 0.18 & 0.04 \\
\hline HMDB10419 & C46:2 TAG & 0.17 & 0.05 & 0.17 & 0.04 & 0.19 & 0.04 \\
\hline HMDB05435 & C50:4 TAG & - & - & 0.17 & 0.04 & - & - \\
\hline $\begin{array}{c}\text { HMDB05405 } \\
\text { Purine } \\
\text { nucleosides }\end{array}$ & C54:3 TAG & - & - & 0.18 & 0.04 & - & - \\
\hline HMDB03331 & 1-methyladenosine & - & - & 0.18 & 0.03 & 0.18 & 0.04 \\
\hline
\end{tabular}

Abbreviations: CE, cholesterol ester; DAG, diacylglycerol; LPC, lysophosphatidylcholine; LPE, lysophosphatidylethanolamine; PC, phosphatidylcholine; TAG, triacylglycerol. Boldface

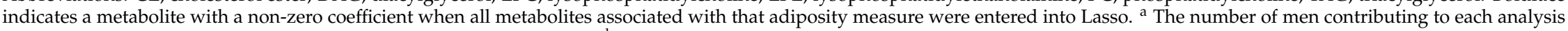
was 234 for BMI, 233 for waist circumference, 219 for derived fat mass. ${ }^{b}$ Representative HMDB IDs provided for PC, DAG, and TAG lipids. ${ }^{c}$ Estimates from partial Pearson correlation, adjusted for age (continuous) and smoking status (ever/never). 


\subsection{Metabolically Defined Obesity and Advanced Prostate Cancer Risk}

Table 3 shows estimated odds ratios for advanced prostate cancer by self-reported or derived adiposity measures and metabolic scores (predicted adiposity given an individual's metabolites). Estimates for all continuously modeled measures were close to null, though confidence intervals were wide. No trends were observed across increasing quartiles of these measures. Estimates were similar in sensitivity analyses excluding men diagnosed in the first two years after the blood draw (data not shown).

Figure 1 shows estimated odds ratios for men cross-classified by self-reported or derived adiposity measures and metabolic scores. For waist circumference and derived fat mass, the estimated odds ratio for advanced prostate cancer was highest among men with a low self-reported or derived adiposity measure but a high metabolic score. However, confidence intervals were wide and overlapping.

Table 3. Estimated odds ratios a for advanced prostate cancers by self-reported or derived adiposity and metabolic scores (predicted adiposity given an individual's metabolites), Health Professionals Follow-Up Study.

\begin{tabular}{|c|c|c|c|c|c|c|}
\hline \multirow{2}{*}{ Adiposity Measure } & \multicolumn{3}{|c|}{ Self-reported or Derived } & \multicolumn{3}{|c|}{ Metabolic Score } \\
\hline & $\begin{array}{l}\text { Cases/ } \\
\text { Total }\end{array}$ & $\begin{array}{l}\text { Odds } \\
\text { Ratio }\end{array}$ & $95 \%$ CI & $\begin{array}{l}\text { Cases/ } \\
\text { Total }\end{array}$ & $\begin{array}{l}\text { Odds } \\
\text { Ratio }\end{array}$ & $95 \% \mathrm{CI}$ \\
\hline \multicolumn{7}{|c|}{ Body mass index $\left(\mathrm{kg} / \mathrm{m}^{2}\right)^{b}$} \\
\hline per $5 \mathrm{~kg} / \mathrm{m}^{2}$ increase & $201 / 409$ & 0.96 & $(0.73,1.27)$ & $201 / 409$ & 1.18 & $(0.57,2.48)$ \\
\hline Quartile 1 & $46 / 104$ & 1.00 & - & $49 / 103$ & 1.00 & - \\
\hline Quartile 2 & $60 / 102$ & 1.78 & $(1.02,3.13)$ & $40 / 102$ & 0.71 & $(0.40,1.23)$ \\
\hline Quartile 3 & $49 / 102$ & 1.14 & $(0.65,1.98)$ & $60 / 102$ & 1.57 & $(0.90,2.75)$ \\
\hline Quartile 4 & $46 / 101$ & 1.04 & $(0.60,1.82)$ & $52 / 102$ & 1.12 & $(0.64,1.97)$ \\
\hline$p$-trend & & & 0.72 & & & 0.20 \\
\hline \multicolumn{7}{|l|}{ Waist circumference $(\mathrm{cm})^{c}$} \\
\hline per $1 \mathrm{SD}$ increase & $200 / 408$ & 0.90 & $(0.73,1.10)$ & $200 / 408$ & 0.99 & $(0.81,1.21)$ \\
\hline Quartile 1 & $53 / 110$ & 1.00 & - & $45 / 102$ & 1.00 & - \\
\hline Quartile 2 & $52 / 96$ & 1.25 & $(0.72,2.19)$ & $52 / 102$ & 1.35 & $(0.77,2.36)$ \\
\hline Quartile 3 & $52 / 110$ & 0.93 & $(0.54,1.60)$ & $53 / 102$ & 1.38 & $(0.79,2.43)$ \\
\hline Quartile 4 & $43 / 92$ & 0.90 & $(0.50,1.59)$ & $50 / 102$ & 1.19 & $(0.67,2.12)$ \\
\hline$p$-trend & & & 0.52 & & & 0.54 \\
\hline \multicolumn{7}{|l|}{ Derived fat mass $(\mathrm{kg})^{\mathrm{d}}$} \\
\hline per 1 SD increase & $193 / 388$ & 0.89 & $(0.73,1.10)$ & $193 / 388$ & 1.07 & $(0.82,1.39)$ \\
\hline Quartile 1 & $49 / 97$ & 1.00 & - & $42 / 97$ & 1.00 & - \\
\hline Quartile 2 & $54 / 97$ & 1.20 & $(0.68,2.13)$ & $51 / 97$ & 1.45 & $(0.82,2.58)$ \\
\hline Quartile 3 & $44 / 97$ & 0.78 & $(0.44,1.38)$ & $54 / 97$ & 1.63 & $(0.92,2.91)$ \\
\hline Quartile 4 & $46 / 97$ & 0.84 & $(0.47,1.50)$ & $46 / 97$ & 1.15 & $(0.64,2.06)$ \\
\hline$p$-trend & & & 0.30 & & & 0.54 \\
\hline
\end{tabular}

Abbreviation: CI, confidence interval. ${ }^{a}$ Estimates from unconditional logistic regression models adjusted for age (years, continuous), history of diabetes (yes/no), physical activity (MET hours/week, continuous), smoking status (ever/never), family history of prostate cancer (yes/no), and recent PSA testing (yes/no). Self-reported/derived adiposity measures and metabolic scores were fit in separate models. ${ }^{b}$ Quartiles of self-reported BMI: [18.6, 23.2], $(23.2,25.2],(25.2,27.5],(27.5,42.4]$. The fourth quartile (101 men, of whom 46 had advanced prostate cancer) includes 45 men with a BMI $>30 \mathrm{~kg} / \mathrm{m}^{2}$, of whom 22 had advanced prostate cancer. Quartiles of BMI metabolic score: [20.8, 24.6], (24.6, 25.5], $(25.5,26.3],(26.3,29.7]$. $^{\text {c }}$ Standard deviation of self-reported waist circumference: $10.1 \mathrm{~cm}$. Standard deviation of waist circumference metabolic score: $3.4 \mathrm{~cm}$. Quartiles of self-reported waist circumference: [73.7, 88.9], (88.9, 94.6], $(94.6,102.0],(102.0,135.0]$. Quartiles of waist circumference metabolic score: [82.7, 92.9], $(92.9,95.3],(95.3,97.2],(97.2,106.0] .{ }^{d}$ Standard deviation of derived fat mass: $6.1 \mathrm{~kg}$. Standard deviation of fat mass metabolic score: $2.9 \mathrm{~kg}$. Quartiles of derived fat mass: [7.9, 17.8], (17.8, 21.3], (21.3, 25.1], (25.1, 45.9]. Quartiles of fat mass metabolic score: [13.7, 19.9], (19.9, 21.7], $(21.7,23.5],(23.5,30.8]$. 
A

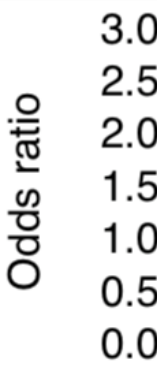

2.5

0.0

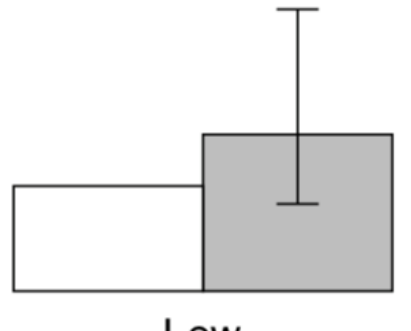

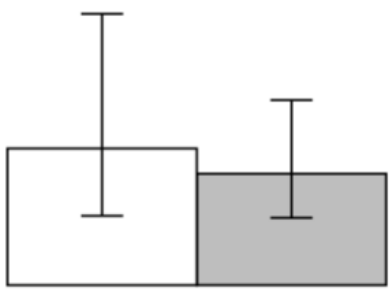

High
Metabolic score for BMI

Low

High

BMI

Median splits of $25 \mathrm{~kg} / \mathrm{m}^{2}$ for BMI and score

B

3.0

$\begin{array}{cl} & 2.5 \\ \text { 을 } & 2.0 \\ \text { 띤 } & 1.5 \\ \text { 응 } & 1.0 \\ \text { 잉 } & 0.5 \\ & 0.0\end{array}$

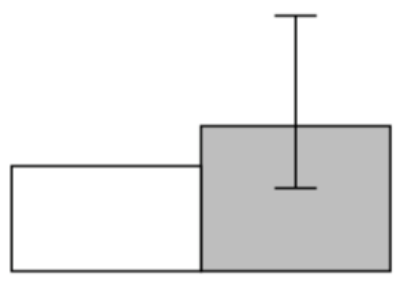

Low

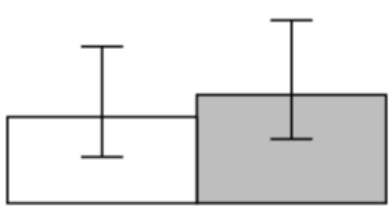

High

Metabolic score

for waist

circumference

Low

High

Waist circumference

Median splits of $95 \mathrm{~cm}$ for waist circumference and score

C

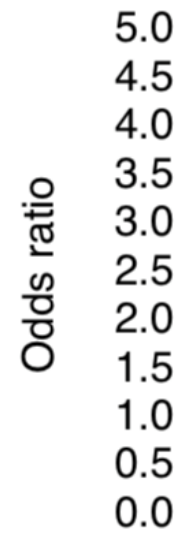

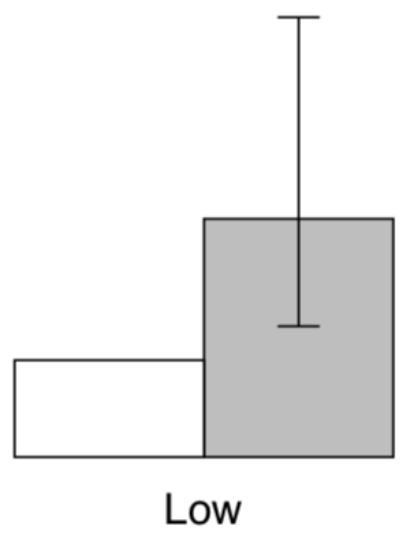

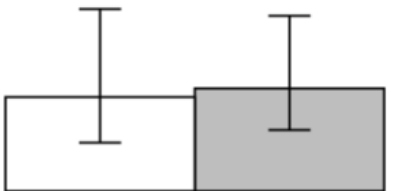

High
Metabolic score for fat mass

\section{Low}

High

\section{Fat mass}

Median splits of $21 \mathrm{~kg}$ for fat mass and score

Figure 1. Odds ratios and 95\% confidence intervals for advanced prostate cancer by (a) BMI-metabolic, (b) waist circumference-metabolic, and (c) fat mass-metabolic groups. Men were classified into one of four groups based on having a self-reported/derived adiposity measure above versus below the median and a metabolic score (predicted adiposity based on metabolites) above versus below the median. Estimates were adjusted for age, history of diabetes, physical activity, smoking status, family history of prostate cancer, and recent PSA testing.

\section{Discussion}

In this analysis of 165 measured metabolites, we identified a substantial number of metabolites associated with BMI, waist circumference, and derived fat mass, with considerable overlap of 
metabolites across adiposity measures. In an independent set of men, neither adiposity measures nor metabolic scores representing metabolic obesity were independently associated with the risk of advanced prostate cancer. However, we found suggestive evidence that men with a low waist circumference or fat mass but high metabolic obesity score were at the highest risk of advanced disease, although the power for these analyses was lower.

Among lipid metabolites, we identified inverse associations for CE, LPC, and LPE, positive associations for DAG and TAG lipid species, and mixed associations for PC with adiposity measures. These findings are largely consistent with a cross-sectional study of 217 metabolites among 2383 Framingham Offspring participants, with the exception of CE and TAG, which showed positive and mixed associations in that study, respectively [4]. That study also found that LPC containing an 18:2 fatty acid was the lipid most strongly associated with BMI and waist circumference [4], in line with our findings. A prospective study identified low LPC 18:2 as a predictor of incident pre-diabetes and diabetes over a seven-year period, independent of BMI [13].

We found that carnitines were positively associated with all adiposity measures. Carnitine, which can be endogenously synthesized or absorbed from dietary sources such as meat, plays an important role in metabolism by transporting long-chain fatty acids across mitochondrial membranes, making them essential for fatty acid $\beta$-oxidation [14,15]. Acylcarnitine accumulation may result from fatty acid oxidation defects in obese and insulin resistant individuals [16]. We found that waist circumference and derived fat mass were associated with two acylcarnitines not associated with BMI: C16 (L-palmitoylcarnitine) and C12:1 (trans-2-dodecenoylcarnitine). A recent cross-sectional study reported that higher levels of these two metabolites may help to distinguish overweight individuals with high versus low visceral fat area ( $\geq 100$ versus $<100 \mathrm{~cm}^{2}$ at L4) [17].

Among amino acids, we identified inverse associations for glutamine and glycine with all adiposity measures, which is consistent with previous findings [4,6-10]. Several studies have also identified positive associations between branched chain amino acids (valine, leucine, and isoleucine) and aromatic amino acids (tyrosine and phenylalanine) with BMI and/or waist circumference [4,6,7,9]. All but isoleucine were included in our study, but we only identified a positive association between BMI and valine when restricting to fasting samples. This may be due to a different distribution of BMI or modifiers, such as diet and physical activity, in our study population compared with others.

Prospective studies have reported associations between glutamine, glycine, and glutamate with future diabetes risk [6,18], and experimental studies have demonstrated that glutamine supplementation in humans and mice leads to improved glucose tolerance $[6,19]$. This suggests that some of these metabolites may not only be biomarkers of obesity but also effectors of its later sequelae. While we found no evidence for an association between metabolically defined obesity and advanced prostate cancer in our study, the identified metabolites may prove relevant for other disease outcomes.

Prior meta-analyses reported an advanced prostate cancer relative risk of 1.09 (95\% CI: 1.02, 1.16; 13 studies) and a prostate cancer-specific mortality relative risk of 1.15 (95\% CI: 1.06, 1.25; 6 studies) per $5 \mathrm{~kg} / \mathrm{m}^{2}$ increase in BMI [1,20]. These estimates are higher than our findings for advanced/fatal prostate cancer (odds ratio 0.96, 95\% CI: 0.73, 1.27), but our power was limited by a small sample. Different estimates across studies may also be related to differences in BMI assessment (i.e., timing, self-reported versus measured), definition of advanced disease, and participant characteristics.

Individuals with the same measured obesity may differ in their underlying metabolic health [2-4], which could be relevant for later disease risk. We took a novel approach to address this by calculating metabolic scores to summarize underlying metabolic obesity. After cross-classifying men by self-reported and metabolically defined obesity, we found that men at the highest risk of advanced prostate cancer had a low adiposity measure but a high metabolic score. The determinants of a normal weight, metabolically obese profile are unknown but may be related to genetic factors influencing adipocyte function, body fat distribution, and insulin resistance and/or lifestyle factors such as physical activity and diet [5,21]. Men in this group may have a strong propensity for dysregulated metabolism, given their unfavorable metabolic factors despite normal weight, which may contribute 
to the development and progression of advanced prostate cancer [22]. Further investigation of this phenotype may provide additional insight into the underlying mechanisms and potential intervention targets for clinically important prostate cancer.

The major strength of our study is the integration of metabolomics with detailed clinical and lifestyle data within a well-characterized prospective cohort. This allowed us to investigate several adiposity measures and many metabolites while adjusting for important covariates. It also allowed us to assess adiposity and metabolites before advanced prostate cancer diagnosis to establish a temporal relationship and eliminate the potential for recall bias.

Our study also has some limitations. We relied on self-reported adiposity measures, which are subject to measurement error. However, a previous validation study in the Health Professionals Follow-up Study (HPFS) showed that self-reported weights and waist circumferences were highly correlated with technician-measured values [23]. We also relied on a single measurement of adiposity and metabolites in midlife, and it is possible that long-term average measures are the most biologically relevant for prostate cancer risk. Nonetheless, a pilot study showed that approximately $90 \%$ of metabolites were reproducible over two years within individuals [24], and we estimated Pearson correlation coefficients $>0.90$ for BMI reports up to six years apart among men in the prostate cancer nested case-control study (data not shown). This suggests that a single measure may be reasonably representative of average values over midlife. Any error in assessing these average values is expected to be independent from the rate of prostate cancer and, therefore, attenuate our estimates. Our power for the analyses of advanced prostate cancer was limited by the sample size. Lastly, our study population consisted of middle-aged or older health professionals of predominantly European ancestry, so our estimates may not be generalizable to other populations with different distributions of adiposity or risk factors.

In summary, we found that BMI, waist circumference, and derived fat mass were associated with a broad range of metabolic alterations, involving lipids, amino acids, and amino acid derivatives. Neither adiposity nor metabolic scores were associated with risk of advanced prostate cancer in this population of men. However, there was suggestive evidence that a subgroup of men with higher measures of metabolic obesity underlying lower measures of waist circumference and fat mass are at a higher risk. The obesity-identified metabolites may inform future integrative-metabolomics research to better identify individuals at the highest risk of disease.

\section{Materials and Methods}

\subsection{Study Population}

The HPFS is an ongoing prospective cohort study of 51,529 US male health professionals aged 40-75 years at enrollment in 1986. The participants reported detailed clinical and lifestyle information at enrollment and every two years thereafter. Blood samples were collected from 18,225 (35\%) participants from 1993-1995. The samples were mailed to our laboratory overnight on cold packs and then centrifuged to collect and store plasma in liquid nitrogen freezers. Participants reported the timing of blood collection and fasting status on a questionnaire returned with the samples.

For the current study, we included participants who had provided a blood sample and been previously selected for nested metabolomic case-control analyses in the HPFS. For the identification of adiposity-associated metabolites, we included 236 controls from case-control analyses of amyotrophic lateral sclerosis (ALS) and Parkinson's disease who had measures of the same metabolites (see also File S1). We selected controls only to minimize the possibility that latent ALS or Parkinson's disease influenced the metabolite or adiposity measures.

To evaluate metabolically defined obesity and advanced prostate cancer risk, we included an independent set of 212 advanced prostate cancer cases (stage T3b/T4/N1/M1 at diagnosis or development of metastasis or death due to prostate cancer during follow-up) and 212 matched controls. The cases were all men diagnosed with advanced prostate cancer between the time of the blood draw and 
September 2010. For each case, one control was selected who was alive and cancer-free at the time of the case's diagnosis. The matching criteria were age ( \pm 1 year), recent PSA testing prior to the blood draw (since January 1, 1992; yes/no), and the time of day, season, and year of blood collection.

Prostate cancer diagnoses were self-reported on biennial questionnaires and verified in a standardized review of medical records and pathology reports. We obtained information on subsequent metastasis from prostate-cancer-specific biennial questionnaires sent to all prostate cancer survivors and their physicians. Prostate-cancer-specific deaths were verified through review of medical records and death certificates.

\subsection{Adiposity Measures and Covariates}

We assessed BMI, waist circumference, and derived fat mass in the questionnaire preceding blood collection. Participants reported their weight and height in 1986 and updated their weight every two years thereafter. They reported their waist circumference in 1987 and 1996. A previous validation study in HPFS showed that self-reported weights and waist circumferences were highly correlated with technician-measured values (Pearson $r=0.97$ and $r=0.95$, respectively) [23]. We derived fat mass using a prediction equation developed in the National Health and Nutrition Examination Survey (NHANES) [25]. Previous validation in NHANES showed that this equation is highly predictive of dual-energy x-ray absorptiometry (DXA)-measured fat mass $\left(R^{2}=0.90\right)$ [25]. We excluded participants missing adiposity measures for the respective analyses (Figure S1). We also excluded one participant with a BMI $>55 \mathrm{~kg} / \mathrm{m}^{2}$ from the BMI analyses to prevent this outlier from affecting our analyses. We assessed cigarette smoking, physical activity, and history of diabetes in the questionnaire before blood collection.

\subsection{Metabolite Profiling}

Plasma metabolites were profiled at the Broad Institute (Cambridge, MA, USA) using the liquid chromatography tandem mass spectrometry (LC-MS) methods described previously (see also File S1) $[24,26]$. A total of 165 known metabolites were analyzed in this study, including lipids (6 cholesterol ester (CE), 11 diacylglycerol (DAG), 8 lysophosphatidylcholine (LPC), 6 lysophosphatidylethanolamine (LPE), 19 phosphatidylcholine (PC), 19 phosphatidylethanolamine (PE), 3 sphingomyelin (SM), and 42 triacylglycerol (TAG)), amino acids, and other small molecules (see File S1 and Figure S2 for details on the metabolite selection). The metabolite peak areas were $l n$-transformed to improve normality and then standardized (to mean $=0, \mathrm{SD}=1$ ) within each project to facilitate analyses across projects.

\subsection{Statistical Analysis}

Among the 236 controls in the ALS and Parkinson's disease studies, we identified metabolites associated with adiposity by calculating partial Pearson correlations between the 165 metabolites and each adiposity measure, adjusting for age and smoking status at the time of the blood draw. We obtained conservative estimates of false discovery rate (FDR) via the Benjamini-Hochberg procedure [27]. We carried forward metabolites with an FDR $p$-value $<0.05$ and $|\mathrm{r}| \geq 0.15$. Lasso regression models were used to further select metabolites for prediction models of metabolic scores, which represented predicted adiposity (the dependent variable) given men's levels of adiposity-associated metabolites (the independent variables). This approach allowed us to account for the relative importance of each identified metabolite in the presence of the others and generate a parsimonious model.

In an independent set of 212 advanced prostate cancer cases and 212 matched controls, we applied these models to predict metabolic scores. For example, the BMI metabolic score ranks men by their expected BMI based on their underlying levels of BMI-associated metabolites. These metabolic scores were not intended to be surrogates of obesity, but rather to provide information about metabolic obesity that may be relevant in the pathophysiology of disease. Their estimation was informed by the relationships between adiposity measures and metabolic profiles observed in the reference population, 
which are shaped by upstream lifestyle and genetic factors. A high metabolic score can be interpreted as having a metabolic profile consistent with an average man of high adiposity in the reference population.

We used logistic regression to estimate odds ratios and $95 \%$ confidence intervals for advanced prostate cancer comparing different levels of each self-reported or derived adiposity measure and metabolic score, fit in separate models. We used unconditional logistic regression for statistical efficiency, adjusting for age, diabetes, physical activity, smoking status, family history of prostate cancer, and recent PSA testing. Additionally adjusting all models for fasting status ( $\geq 8$ versus $<8 \mathrm{~h}$ ) and adjusting fat mass models for height did not influence our estimates (data not shown).

\subsubsection{Subgroup Analyses}

We estimated the risk for men cross-classified by their self-reported or derived adiposity measures and metabolic scores, each dichotomized at its median.

\subsubsection{Sensitivity Analyses}

We repeated the analyses to identify adiposity-associated metabolites after restricting to men with (1) complete information on all adiposity measures and (2) fasting blood samples. We repeated the analyses for advanced prostate cancer after excluding men diagnosed in the first two years after the blood draw to minimize the chance that latent disease influenced their metabolite or adiposity measures.

The study protocol was approved by the institutional review boards of the Brigham and Women's Hospital and Harvard T.H. Chan School of Public Health, and those of participating registries as required. The analyses were conducted using $\mathrm{R}$, version 3.6.0, and SAS, version 9.4 (SAS Institute, Inc. Cary, NC, USA).

Supplementary Materials: The following are available online at http://www.mdpi.com/2218-1989/10/3/99/s1, File S1. Detail on metabolite profiling, excluded metabolites, included metabolites, File S2. Lasso models used for predicting adiposity using associated metabolites, Figure S1. Flowchart of participant selection, Figure S2. Flowchart of metabolite selection, Figure S3. Overlap in identified metabolites across adiposity measures (from Main Table 2), Table S1. Metabolites associated with adiposity measures (FDR $p$-value $<0.05$ and $|r| \geq 0.15$ ) among 217 controls from nested case-control studies with data on all three adiposity measures, Health Professionals Follow-Up Study, 1993-1996, Table S2. Metabolites associated with adiposity measures (FDR $p$-value $<0.05$ and $|\mathrm{r}|$ $\geq 0.15)$ among controls $(n=118-126)^{\text {a }}$ from nested case-control studies with fasting $(\geq 8 \mathrm{~h})$ blood samples, Health Professionals Follow-Up Study, 1993-1996.

Author Contributions: Conceptualization, B.A.D, E.M.E., and L.A.M.; methodology, B.A.D, E.M.E., B.C.H., and L.A.M.; formal analysis, B.A.D., and C.B.C.; writing-original draft preparation, B.A.D., E.M.E., and L.A.M.; writing-review and editing, B.A.D, E.M.E., B.C.H., K.M.W., A.H.E., A.A., C.H.P., O.A.Z., M.G.V.H., C.B.C., E.G., and L.A.M.; visualization, B.A.D.; supervision, L.A.M.; project administration, L.A.M.; funding acquisition, A.A., and L.A.M. All authors have read and agreed to the published version of the manuscript.

Funding: This project was supported by funding from the Dana-Farber/Harvard Cancer Center Specialized Program of Research Excellence (SPORE) in Prostate Cancer (P50 CA090381). B.A.D. is supported by an ASISA Fellowship. L.A.M. and K.M.W. are supported by Young Investigator Awards from the Prostate Cancer Foundation. A.A. receives research support from the NIH (R01 NS045893, R01 NS089619). The HPFS is supported by the NIH/NCI U01 CA167552.

Acknowledgments: We would like to thank the participants and staff of the Health Professionals Follow-Up Study for their valuable contributions as well as the following state cancer registries for their help: AL, AZ, AR, CA, CO, CT, DE, FL, GA, ID, IL, IN, IA, KY, LA, ME, MD, MA, MI, NE, NH, NJ, NY, NC, ND, OH, OK, OR, PA, RI, SC, TN, TX, VA, WA, and WY. The authors assume full responsibility for analyses and interpretation of these data.

Conflicts of Interest: The authors declare no conflict of interest. The funders had no role in the design of the study; the collection, analyses, or interpretation of data; the writing of the manuscript; or in the decision to submit the manuscript for publication.

\section{References}

1. Cao, Y.; Ma, J. Body mass index, prostate cancer-specific mortality, and biochemical recurrence: A systematic review and meta-analysis. Cancer Prev Res. 2011, 4, 486-501. [CrossRef] [PubMed]

2. Stefan, N.; Schick, F.; Haring, H.U. Causes, characteristics, and consequences of metabolically unhealthy normal weight in humans. Cell Metab. 2017, 26, 292-300. [CrossRef] [PubMed] 
3. Batch, B.C.; Shah, S.H.; Newgard, C.B.; Turer, C.B.; Haynes, C.; Bain, J.R.; Muehlbauer, M.; Patel, M.J.; Stevens, R.D.; Appel, L.J.; et al. Branched chain amino acids are novel biomarkers for discrimination of metabolic wellness. Metabolism. 2013, 62, 961-969. [CrossRef]

4. Ho, J.E.; Larson, M.G.; Ghorbani, A.; Cheng, S.; Chen, M.H.; Keyes, M.; Rhee, E.P.; Clish, C.B.; Vasan, R.S.; Gerszten, R.E.; et al. Metabolomic profiles of body mass index in the Framingham Heart Study reveal distinct cardiometabolic phenotypes. PLOS ONE. 2016, 11, e0148361. [CrossRef] [PubMed]

5. Wildman, R.P.; Muntner, P.; Reynolds, K.; McGinn, A.P.; Rajpathak, S.; Wylie-Rosett, J.; Sowers, M.R. The obese without cardiometabolic risk factor clustering and the normal weight with cardiometabolic risk factor clustering: Prevalence and correlates of 2 phenotypes among the US population (NHANES 19992-004). Arch. Intern. Med. 2008, 168, 1617-1624. [CrossRef] [PubMed]

6. Cheng, S.; Rhee, E.P.; Larson, M.G.; Lewis, G.D.; McCabe, E.L.; Shen, D.; Palma, M.J.; Roberts, L.D.; Dejam, A.; Souza, A.L.; et al. Metabolite profiling identifies pathways associated with metabolic risk in humans. Circulation. 2012, 125, 2222-2231. [CrossRef]

7. Moore, S.C.; Matthews, C.E.; Sampson, J.N.; Stolzenberg-Solomon, R.Z.; Zheng, W.; Cai, Q.; Tan, Y.T.; Chow, W.H.; Ji, B.T.; Liu, D.K.; et al. Human metabolic correlates of body mass index. Metabolomics. 2014, 10, 259-269. [CrossRef]

8. Moore, S.C.; Playdon, M.C.; Sampson, J.N.; Hoover, R.N.; Trabert, B.; Matthews, C.E.; Ziegler, R.G. A metabolomics analysis of body mass index and postmenopausal breast cancer risk. J. Natl Cancer Inst. 2018, 110, 588-597. [CrossRef]

9. Newgard, C.B.; An, J.; Bain, J.R.; Muehlbauer, M.J.; Stevens, R.D.; Lien, L.F.; Haqq, A.M.; Shah, S.H.; Arlotto, M.; Slentz, C.A.; et al. A branched-chain amino acid-related metabolic signature that differentiates obese and lean humans and contributes to insulin resistance. Cell Metab. 2009, 9, 311-326. [CrossRef]

10. Ottosson, F.; Brunkwall, L.; Ericson, U.; Nilsson, P.M.; Almgren, P.; Fernandez, C.; Melander, O.; Orho-Melander, M. Connection between BMI-related plasma metabolite profile and gut microbiota. J. Clin Endocrinol Metab. 2018, 103, 1491-1501. [CrossRef]

11. Boulet, M.M.; Chevrier, G.; Grenier-Larouche, T.; Pelletier, M.; Nadeau, M.; Scarpa, J.; Prehn, C.; Marette, A.; Adamski, J.; Tchernof, A. Alterations of plasma metabolite profiles related to adipose tissue distribution and cardiometabolic risk. Am. J. Physiol Endocrinol Metab. 2015, 309, E736-E746. [CrossRef] [PubMed]

12. Foerster, J.; Hyotylainen, T.; Oresic, M.; Nygren, H.; Boeing, H. Serum Lipid and Serum Metabolite Components in relation to anthropometric parameters in EPIC-Potsdam participants. Metabolism. 2015, 64, 1348-1358. [CrossRef] [PubMed]

13. Wang-Sattler, R.; Yu, Z.; Herder, C.; Messias, A.C.; Floegel, A.; He, Y.; Heim, K.; Campillos, M.; Holzapfel, C.; Thorand, B.; et al. Novel biomarkers for pre-diabetes identified by metabolomics. Mol. Syst. Biol. 2012, 8, 615. [CrossRef] [PubMed]

14. Rebouche, C. Carnitine. In Modern Nutrition in Health and Disease, 10th ed.; Lippincott, Williams \& Wilkins: Philadelphia, PA, USA, 2006; pp. 537-544.

15. Rebouche, C.J. Kinetics, pharmacokinetics, and regulation of L-carnitine and acetyl-L-carnitine metabolism. Ann. NY Acad Sci. 2004, 1033, 30-41. [CrossRef]

16. Mihalik, S.J.; Goodpaster, B.H.; Kelley, D.E.; Chace, D.H.; Vockley, J.; Toledo, F.G.; DeLany, J.P. Increased levels of plasma acylcarnitines in obesity and type 2 diabetes and identification of a marker of glucolipotoxicity. Obesity 2010, 18, 1695-1700. [CrossRef]

17. Baek, S.H.; Kim, M.; Kim, M.; Kang, M.; Yoo, H.J.; Lee, N.H.; Kim, Y.H.; Song, M.; Lee, J.H. Metabolites distinguishing visceral fat obesity and atherogenic traits in individuals with overweight. Obesity 2017, 25, 323-331. [CrossRef]

18. Floegel, A.; Stefan, N.; Yu, Z.; Muhlenbruch, K.; Drogan, D.; Joost, H.G.; Fritsche, A.; Haring, H.U.; Hrabe de Angelis, M.; Peters, A.; et al. Identification of serum metabolites associated with risk of type 2 diabetes using a targeted metabolomic approach. Diabetes 2013, 62, 639-648. [CrossRef]

19. Greenfield, J.R.; Farooqi, I.S.; Keogh, J.M.; Henning, E.; Habib, A.M.; Blackwood, A.; Reimann, F.; Holst, J.J.; Gribble, F.M. Oral glutamine increases circulating glucagon-like peptide 1, glucagon, and insulin concentrations in lean, obese, and type 2 diabetic subjects. Am. J. Clin Nutr. 2009, 89, 106-113. [CrossRef]

20. Discacciati, A.; Orsini, N.; Wolk, A. Body mass index and incidence of localized and advanced prostate cancer-a dose-response meta-analysis of prospective studies. Ann. Oncol. 2012, 23, 1665-1671. [CrossRef] 
21. Scott, R.A.; Fall, T.; Pasko, D.; Barker, A.; Sharp, S.J.; Arriola, L.; Balkau, B.; Barricarte, A.; Barroso, I.; Boeing, H.; et al. Common genetic variants highlight the role of insulin resistance and body fat distribution in type 2 diabetes, independent of obesity. Diabetes 2014, 63, 4378-4387. [CrossRef]

22. Giovannucci, E.; Michaud, D. The role of obesity and related metabolic disturbances in cancers of the colon, prostate, and pancreas. Gastroenterology 2007, 132, 2208-2225. [CrossRef] [PubMed]

23. Rimm, E.B.; Stampfer, M.J.; Colditz, G.A.; Chute, C.G.; Litin, L.B.; Willett, W.C. Validity of self-reported waist and hip circumferences in men and women. Epidemiology 1990, 1, 466-473. [CrossRef]

24. Townsend, M.K.; Clish, C.B.; Kraft, P.; Wu, C.; Souza, A.L.; Deik, A.A.; Tworoger, S.S.; Wolpin, B.M. Reproducibility of metabolomic profiles among men and women in 2 large cohort studies. Clin Chem. 2013, 59, 1657-1667. [CrossRef] [PubMed]

25. Lee, D.H.; Keum, N.; Hu, F.B.; Orav, E.J.; Rimm, E.B.; Sun, Q.; Willett, W.C.; Giovannucci, E.L. Development and validation of anthropometric prediction equations for lean body mass, fat mass and percent fat in adults using the National Health and Nutrition Examination Survey (NHANES) 1999-2006. Br. J. Nutr. 2017, 118, 858-866. [CrossRef] [PubMed]

26. Paynter, N.P.; Balasubramanian, R.; Giulianini, F.; Wang, D.D.; Tinker, L.F.; Gopal, S.; Deik, A.A.; Bullock, K.; Pierce, K.A.; Scott, J.; et al. Metabolic predictors of incident coronary heart disease in women. Circulation 2018, 137, 841-853. [CrossRef] [PubMed]

27. Benjamini, Y.; Hochberg, Y. Controlling the false discovery rate: A practical and powerful approach to multiple testing. J. R. Statist. Soc. B 1995, 57, 289-300. [CrossRef]

(C) 2020 by the authors. Licensee MDPI, Basel, Switzerland. This article is an open access article distributed under the terms and conditions of the Creative Commons Attribution (CC BY) license (http://creativecommons.org/licenses/by/4.0/). 